\title{
Mémoire(s) et territoire(s) : les bulletins municipaux de Villeurbanne
}

Memory(ies) and Territory(ies): Examining Municipal Reports in Villeurbanne (France)

Julien Auboussier et Isabelle Garcin-Marrou

\section{OpenEdition Journals}

Édition électronique

URL : http://journals.openedition.org/edc/2948

DOI : 10.4000/edc. 2948

ISSN : 2101-0366

Éditeur

Université Lille-3

Édition imprimée

Date de publication : 1 décembre 2011

Pagination : 47-62

ISBN : 978-2-917562-06-2

ISSN : $1270-6841$

Référence électronique

Julien Auboussier et Isabelle Garcin-Marrou, « Mémoire(s) et territoire(s) : les bulletins municipaux de Villeurbanne », Études de communication [En ligne], 37 | 2011, mis en ligne le 01 décembre 2013, consulté le 30 avril 2019. URL : http://journals.openedition.org/edc/2948 ; DOI : 10.4000/edc.2948

Ce document a été généré automatiquement le 30 avril 2019

(c) Tous droits réservés 


\title{
Mémoire(s) et territoire(s) : les bulletins municipaux de Villeurbanne
}

\author{
Memory(ies) and Territory(ies): Examining Municipal Reports in Villeurbanne \\ (France)
}

Julien Auboussier et Isabelle Garcin-Marrou

1 «Ce n'est pas par mégarde que nous disons de ce qui est advenu qu'il a eu lieu » notait Paul Ricœur (Ricœur, 2000, 49). La mémoire s'ancre en effet dans l'espace et participe à la construction des territoires. Le cadre spatial constitue un repère stabilisé dans le temps qui favorise le souvenir en même temps qu'il porte, lui-même, les traces, plus ou moins visibles, de l'histoire du groupe (Halbwachs, 1950 ; Haas, Jodelet, 2000). L'espace urbain, dans sa distribution, ses formes, sa consistance, constitue ainsi un support et un symbole du passé de la communauté et, par là même, un vecteur important de son identité et de sa mémoire. Comment la mémoire contribue-t-elle à l'identité de la ville, à la différenciation et à l'autonomie du territoire urbain? De quelle façon alimente-t-elle le récit et les imaginaires de la ville? Comment investit-elle des lieux spécifiques de la ville et, ce faisant, leur offre-t-elle une consistance symbolique? Quelles sont les tensions qui animent cette mémoire discursive de la ville ? C'est à partir de ces questions que nous proposons d'évaluer la place de la mémoire dans la construction territoriale d'un espace urbain, et ce à partir d'un médiateur privilégié de l'identité urbaine: les bulletins municipaux (BM) et, plus spécifiquement, ceux de la ville de Villeurbanne, cité d'environ 120000 habitants de l'est lyonnais.

2 Les pistes de réflexion ici proposées reposent sur une analyse exhaustive - thématique et narrative - des éditoriaux et articles des rubriques « Histoires vécues » et " Mémoire » publiés dans le BM depuis $1974^{1}$. Il s'agit de repérer quels sont les mots et les figures à partir desquels se construit le grand récit de la ville dans les BM. 


\section{Mémoire et territoire : l'impératif constructiviste}

\section{De l'espace au territoire}

3 Un nombre considérable de projets à vocation locale participe à la valorisation culturelle de la mémoire des territoires. Les usages des politiques et projets culturels sont souvent peu interrogés et relèvent d'un essentialisme qui masque les processus de construction symbolique dont la mémoire et le territoire - et les représentations collectives qui les alimentent - sont issus. L'affirmation identitaire du territoire y relève le plus souvent de l'incantation performative.

4 La géographie sociale a précisément pensé, à partir des années 1990, l'imbrication de l'espace et des rapports sociaux, et les enjeux identitaires que cette relation soulève. Ainsi, pour Guy Di Meo, «la notion de territoire exprime une appropriation sociale de l'espace géographique par des groupes qui se donnent une représentation identitaire. Bien que construit sur un canevas de lieux concrets à fort contenu symbolique, le territoire s'affirme surtout en tant qu'artefact, représentations sociales et idéologie » (Di Meo, 2000, 320). Il apparaît à l'articulation du vécu et du social, de l'individuel et du collectif, de l'expérience et du politique. En fait, tout espace peut devenir territoire à condition que l'un et l'autre soient " pris dans un rapport social de communication » (Raffestin, 1980, 132). C'est pourquoi le territoire relève d'une construction sociale dynamique dont la configuration ne peut pas être pensée à l'écart des enjeux sociopolitiques et culturels qui parcourent la communauté. L'impératif constructiviste s'impose donc lorsqu'on aborde les notions de territoire et de mémoire.

\section{Ville, information, communication}

5 Si le recours aux sciences de l'information et de la communication est pertinent pour penser l'espace urbain, c'est, premièrement, que la ville est un lieu social et un lieu de médiation qui naît de l'échange et donc de la communication ; c'est, deuxièmement, que la ville constitue un espace d'information «au sein duquel s'élabore, se diffuse et s'interprète l'information et comme un espace qui existe grâce à l'information produite sur lui. La consistance réelle, physique de la ville est, en quelque sorte, subsumée par la consistance symbolique qu'en donnent les médias, les discours et les représentations » (Lamizet, 2002, 18-19). A ce titre, l'espace urbain est un lieu fort d'investissement en termes de discours, de représentations, de stéréotypes, d'images. L'information propose une modalité du réel lisible et perceptible. C'est dire l'importance des stratégies de communication et des modalités par lesquelles la ville se donne à voir et se raconte. Au sein de la communication territoriale, le BM constitue un médiateur privilégié de l'identité d'une ville.

\section{Bulletin municipal : les enjeux du discours}

Isabelle Pailliart relève trois dimensions du journal municipal: la communication des services locaux, la communication des acteurs du territoire, la communication de l'institution politique (Pailliart, 2006, 118-119). Ces trois registres de communication alimentent une visée commune : la valorisation du territoire. Les BM s'inscrivent au cœur 
des stratégies de mise en scène de la ville adoptées par l'équipe municipale et contribuent à la réactivation incessante du territoire. Dans un espace urbain objectivement éclaté, dans un espace où se déploient d'innombrables identités, les BM visent à maintenir « la fiction d'un espace clairement délimité, non problématique [...] en euphémisant les antagonismes 'objectifs' »(Le Bart, 2000, 179). Une part essentielle des stratégies identitaires de la municipalité s'attache ainsi à la configuration de cet insaisissable "nous", condition à une vision commune de la cité. Ainsi, depuis 1974, le BM de Villeurbanne débute par l'éditorial du maire et un "nous» d'ouverture dans lequel l'ensemble des habitants est invité à s'identifier. Le maire s'y présente comme le garant des intérêts de la commune et de ses habitants et comme le représentant des valeurs censées être partagées par les habitants. En proposant un ensemble de représentations à partager, le BM donne un sens à la ville.

7 Ancien faubourg de Lyon, Villeurbanne est aujourd'hui la plus grande ville de la périphérie lyonnaise. Située à l'est de la métropole, la cité, issue des mutations industrielles, a longtemps constitué la banlieue ouvrière de Lyon. Elle compte seulement 4500 habitants en 1851, 41700 en 1911, dépasse les 100000 en 1962. Au dernier recensement, en 2006, Villeurbanne compte 138596 habitants. Après les ouvriers des départements limitrophes, les usines villeurbannaises attirent dès le dernier quart du XIX ${ }^{\mathrm{e}}$ siècle de nombreux travailleurs italiens, la plupart ouvriers du bâtiment. A l'aube du XX siècle, les Italiens sont rejoints par les Espagnols puis les Algériens dans les années 20. Ce sont aussi des Arméniens qui fuient les massacres. En 1931, $17 \%$ de la population villeurbannaise est étrangère. De nombreux Tunisiens et Portugais viennent ensuite grossir la masse des travailleurs. Issue des mutations industrielles et du brassage des populations, Villeurbanne est ancrée à gauche depuis la fin de la Seconde Guerre mondiale comme l'illustre l'inscription politique des maires qui se sont succédé depuis un demi-siècle ${ }^{2}$. La ville doit longtemps faire face aux prétentions hégémoniques de sa grande voisine. Ainsi tout au long de la seconde moitié du XIX ${ }^{\mathrm{e}}$ siècle, plusieurs tentatives d'annexion échouent. La dernière et sérieuse tentative a lieu en 1903. Avec l'arrivée d'Edouard Herriot à la mairie de Lyon en 1905, les prétentions d'annexion s'estompent. Pour autant, la relation avec Lyon continue à alimenter, au cours du XX siècle, la revendication identitaire de Villeurbanne.

\section{L'opposition à Lyon comme première modalité de l'affirmation identitaire}

Quand Villeurbanne se raconte, la rivalité à Lyon apparait longtemps comme un fort vecteur identitaire. Dans les éditoriaux de Charles Hernu (maire de 1977 à 1990), elle apparaît très clairement comme le mode configurant de la revendication identitaire de la cité. S'affirmer face à Lyon, c'est rejeter l'étiquette stigmatisante de "banlieue » et revendiquer celle de "ville-centre". C'est que, à partir de 1985 à peu près, le mot banlieue « va servir à désigner tout à la fois les lieux, les maux et les peurs associés à la crise sociale et politique née du chômage et du racisme actuels" (Faure, 2010). La banlieue apparaît comme la tumeur maligne de la ville centre: Le Monde titre ainsi le 23 mars 1986 "L'agglomération lyonnaise est malade de ses banlieues ». Qui dit banlieue ne parle donc pas forcément d'espace géographique. Les mots sont porteurs de domaines de mémoire et dans le mot «banlieue » résonnent, dans une perspective dialogique, les discours passés et présents (futurs aussi), s'y reflète aussi un faisceau d'images, souvent 
anxiogènes. Refuser le stigmate passe alors par l'affirmation et la valorisation de l'autonomie de la ville. Ainsi, l'indépendance de la cité apparaît comme un leitmotiv dans les éditoriaux de Charles Hernu. En 1979 : «Villeurbanne est à côté de Lyon, enfin de l'autre côté du Rhône. Elle fut et elle est cité ouvrière, ville de travail. Elle ne se considère pas comme une banlieue et si la Cité doit être gérée, animée en tenant compte d'une nécessaire harmonie avec la gestion et l'animation de Lyon, pour autant la ville est différente, autre » [éditorial, mars 1979]. En 1981 : Villeurbanne est « fière de ce qu'elle est; proche de Lyon certes et pourtant différente, par son histoire, sa vie des quartiers, son caractère populaire » [éditorial, février 1981]. En 1987, Charles Hernu revient sur ses dix premières années de mandat : "Une seule volonté a animé les équipes qui ont travaillé avec moi : changer la ville pour changer la vie. Et ce défi a été relevé. Villeurbanne est une grande cité, reconnue. Elle a perdu son statut de banlieue terne et où rien ne se passe. Au contraire, elle provoque des jalousies» [éditorial mars 1987]. Quelques mois après : "Villeurbanne est aujourd'hui une grande ville reconnue. Cette simple banlieue ouvrière, laborieuse et populaire est devenue une ville à part entière [...] Villeurbanne est à même de jouer pleinement son rôle. Au côté de Lyon » [éditorial, avril 1987]. L'année suivante : « Villeurbanne est une cité dont chacun sait qu'elle n'est pas une banlieue mais une ville authentique avec ses quartiers, ses centres, ses 23 parcs d'activité économique, son TNP, son équipe fanion de basket [...] son campus universitaire avec ses 16000 étudiants, ses 2000 commerçants et artisans, ses 800 entreprises » [éditorial, octobre 1988].

Dans les éditoriaux de Charles Hernu, l'histoire de Villeurbanne est l'histoire d'une émancipation, autrement dit, d'une distinction territoriale. Par la voix de son maire, garant de l'illusoire "nous » communautaire, Villeurbanne raconte la conquête de son autonomie.

10 Après le décès de Charles Hernu et l'arrivée de Gilbert Chabroux à la tête de la municipalité en 1990, la rivalité avec Lyon tend à s'effacer; ce que le développement de l'intercommunalité contribue à expliquer. Franck Scherrer note d'ailleurs que «l'histoire du maillage intercommunal lyonnais ne commence pas avec la création de la Communauté Urbaine en 1970, mais débute sans doute avec... l'échec de l'annexion de Villeurbanne par Lyon au début de ce siècle » (Scherrer, 1995, 105). La rivalité à Lyon comme mode configurant de l'affirmation identitaire n'apparait aujourd'hui plus que sur un mode anecdotique ou sur un mode historique quand il s'agit de revenir sur les multiples tentatives d'annexion de Villeurbanne par Lyon au cours du XIX ${ }^{e}$ siècle. Ces dernières constituent en quelque sorte la pré-histoire de Villeurbanne à laquelle l'édification du centre-ville en 1934 mettra fin.

\section{Centre-ville et quartiers : un investissement mémoriel différencié}

11 Le concept de ville s'organise notamment autour de l'opposition centre/périphérie (Lamizet, 1997, 39). Au cours des années 1990, avec le développement des relations nouées dans le cadre de l'intercommunalité, le modèle centre/périphérie est beaucoup moins mobilisé pour penser la relation à Lyon que pour penser Villeurbanne. Sous les mandats de Gilbert Chabroux (1990-2001), la distinction centre/périphérie se déplace de la métropole lyonnaise (ville centre/banlieue) au territoire villeurbannais. La distinction, essentiellement spatiale, vise alors à distinguer géographiquement le centre-ville des quartiers périphériques. Dans le BM, la distinction contribue également à configurer deux 
modes d'appréhension de l'espace et des territoires urbains. Ainsi, l'information municipale est parcourue par la tension qui s'exerce entre le désir de faire territoire (mémoire singulière du territoire) et la volonté de reconnaître aux quartiers leurs spécificités et, ce faisant, admettre la fragmentation de l'espace urbain (mémoire multiple du territoire).

\section{L'investissement mémoriel du centre-ville : récit fondateur et figure du pionnier}

$\mathrm{Si}$, avec les logiques institutionnelles propres à la métropolisation, le contexte d'opposition à Lyon ne constitue plus la pierre angulaire de l'affirmation identitaire villeurbannaise après 1990, il reste pourtant important au sens où il contribue à alimenter et à justifier l'investissement mémoriel du centre-ville. Le quartier des Gratte-Ciel est un ensemble architectural composé de deux tours monumentales, de l'avenue HenriBarbusse et de l'hôtel de ville qui clôt la perspective. Construit sous le mandat de Lazare Goujon et inauguré en 1934, il apparaît comme une œuvre d'avant-garde issue de la volonté politique de la municipalité. Villeurbanne est alors une grande cité ouvrière de 80000 habitants et l'objectif prioritaire est de proposer aux travailleurs des logements décents qui répondent aux nouvelles exigences sanitaires. Il s'agit par ailleurs d'offrir un véritable centre-ville, de créer de la centralité dans un espace jusqu'alors fragmenté. La construction du quartier relève de l'idéologie dans le sens où elle repose sur l'idée que l'on peut transformer les rapports sociaux par l'aménagement urbain (Bonneville, 1978). Aujourd'hui, les commentateurs s'accordent également sur l'enjeu identitaire d'un tel projet : l'urbain pour résister à l'hégémonie de Lyon: "Les Gratte-Ciel mettent aussi en évidence le rôle primordial de l'architecture dans la construction d'une identité et posent la question de la gestion de la ville par le politique. On peut légitimement se demander si, sans cet ensemble construit pour affirmer l'identité villeurbannaise face aux tendances hégémoniques de Lyon, Villeurbanne en tant que commune indépendante existerait toujours » (Clémençon, 2004, 15). La force évocatrice des Gratte-Ciel tient sans doute à la possibilité d'embrasser du regard le tryptique architectural (tours, hôtel de ville, avenue) : « les lieux les plus remarquables s'embrassent du regard »(Di Meo, 1998, 39).

13 Au sein de la communication municipale, le récit de la ville trouve dans l'édification des Gratte-Ciel l'acte fondateur de la cité. S'il existe une pré-histoire de la ville, Villeurbanne entre bien dans la modernité en 1934 quand le quartier est inauguré. Ce récit des origines est très largement repris dès que Villeurbanne pense son histoire. Pour la rénovation des deux tours en 2000, un dossier intitulé «Gratte-ciel: patrimoine très humain » est proposé dans le BM. Les Gratte-Ciel apparaissent comme un vecteur de continuité entre le passé et le présent: "L'histoire a donné au quartier des Gratte-Ciel un aspect mythique de centre-ville organisé à partir de l'avenue Henri Barbusse, espace vivant et monumental [...]. Répondant aux préoccupations sociales et politiques des années 30, cet ensemble architectural a fait l'objet d'une appropriation et d'une identification telles qu'il symbolise encore au XXI' siècle la ville toute entière » [février 2000]. En 2003, dans un dossier intitulé « Un certain regard » et proposant une sorte d'inventaire du patrimoine villeurbannais, l'éditorial précise: « Même si la Villa Urbana revendique sa trace 50 ans avant J.-C., elle a affirmé son identité en plantant ses Gratte-Ciel dans les années 30 dressant ce centre-ville contre toute tentation et tentative d'annexion par la ville centre voisine. Cour contre cœur, et parfois dent contre dent, 
Villeurbanne va se payer l'audace d'une ville sans bourgeoisie, sans conservatisme " [éditorial, mars 2003].

Le contexte politique dans lequel apparaît et auquel répond la construction des GratteCiel, l'imaginaire que celui-ci mobilise alors, tout concorde à faire du centre-ville un lieu symbolique fort. Et l'on constate un investissement mémoriel de cet espace qui répond à une volonté de tisser du lien entre, d'une part, l'avant-gardisme de Lazare Goujon et, d'autre part, l'identité de la ville actuelle et l'action de l'équipe municipale. Le récit des origines ne peut être que mythique et politique : «il ne saurait résulter d'une succession d'événements singuliers dont on pourrait faire la chronologie mais, au contraire, il s'agit d'une histoire politique, c'est-à-dire d'une histoire dont la crédibilité et la légitimité ne tiennent pas à sa conformité à un réel que l'on peut connaître mais à l'adhésion de ceux qui, voyant leur propre histoire dans ce discours, le reconnaissent comme médiation ». (Lamizet, 1997, 25). Le récit de la Villeurbanne moderne est un récit figé dans les discours institutionnels, parfaitement calibré, toujours disponible. Il s'appuie notamment sur la valorisation de Lazare Goujon, figure du "pionnier», qui participe à la construction sociale du récit. Les logiques sociales, politiques, économiques complexes qui conduisent à l'édification des Gratte-Ciel disparaissent ainsi au profit de la valorisation d'un homme et de sa volonté. Le récit se fige autour de la personne du maire: "La spécificité [de Villeurbanne], tant par rapport à sa grande voisine qu'aux cités de la banlieue industrielle, est aujourd'hui bien affirmée. Alain Gilles, avec l'Asvel, Roger Planchon avec le TNP, en constituent les fleurons les plus connus. Néanmoins, quels que soient leurs talents [...], Villeurbanne ne serait sans doute pas ce qu'elle est aujourd'hui sans le parti pris urbanistique de Lazare Goujon » [éditorial, juin 1984] ; «Au début du siècle, Villeurbanne n'était pas vraiment une ville [...]. On s'enfonçait alors dans la boue et on vivait mal dans des pièces sans eau, sans confort. Rien de bien gai donc mais un homme allait changer le cours des choses. Elu maire en 1924, le Dr Lazare Goujon, socialiste, décida que Villeurbanne devait s'imposer en tant que commune indépendante, susceptible de rivaliser avec Lyon. Un vaste programme de construction allait donner naissance au quartier des Gratte-Ciel [...]. Le XX ${ }^{\mathrm{e}}$ siècle s'installait à Villeurbanne. Lazare Goujon avait gagné son pari » [Gratte-Ciel 1934. Une ville est née, juin 1994].

Un espace urbain devient signifiant et jouit d'un capital symbolique à condition d'être l'objet de discours. "La mémoire d'un lieu, c'est avant tout la somme des discours produits à son égard» (Vadelorge, 2003, 7). Les cérémonies d'anniversaire du quartier sont autant d'occasions de répéter la "grande histoire» de Villeurbanne. C'est que la construction du territoire se nourrit de l'épaisseur du temps et de la répétition. Les cérémonies participent ainsi à l'actualisation de la singularité sociale et politique de l'histoire et de la mémoire villeurbannaises. Elles constituent de véritables moments discursifs : au-delà du BM, colloques, publications, recueils de témoignages participent à l'actualisation de la mémoire des Gratte-Ciel, et par métonymie, à celle de Villeurbanne.

Le récit des Gratte-Ciel encourage le ralliement communautaire et les opportunités offertes par les événements culturels constituent des occasions de mettre en scène, notamment par la photographie, l'appropriation du centre-ville par la communauté. Au cours de ces événements, la ville se donne à voir « avec la distanciation de la médiation esthétique » (Lamizet, 2011, 180). Surtout, le BM relaye l'élan collectif qui amène, dans la ville-événement (Boullier, 2010), l'ensemble de la communauté - notamment «des quartiers » - à se retrouver dans le centre.

Dans la presse locale, la photographie participe à la construction d'une identité singulière de la ville susceptible d'entretenir ou d'affirmer un sentiment d'appartenance (Lussagnet, 
1995, 85). Au cours des années 1970, la une du BM propose la photographie d'un lieu identifiable : place, rue, immeubles, etc. Tous les espaces de la ville, même périphériques, sont alors susceptibles d'offrir leur esthétique à la mise en image de la cité. L'on offre ainsi aux lecteurs la possibilité de reconnaître un fragment de la ville. Dès le début des années 1980, dans un contexte de professionnalisation des services chargés de la communication, le bulletin voit ses unes largement évoluer. La simple photographie d'un espace de la ville jouant exclusivement sur l'effet de réel et la représentation d'un espace vécu disparaît. La grande majorité des photographies est alors achronique, située en dehors du temps et n'offre plus la possibilité de constater les effets du temps, l'évolution des espaces, etc. En fait, depuis 1981, un lieu, un seul, est très régulièrement mobilisé et reste identifiable en une : la perspective Gratte-Ciel/Hôtel de ville/avenue Henri-Barbusse.

Après 1989, les deux tours des Gratte-Ciel offrent même leur silhouette à la consistance esthétique de la cité en intégrant la signature officielle de la ville. Elles s'imposent sous le régime de la synecdoque comme le symbole de la ville, comme le signe de la ville et s'affirment ainsi comme une ressource pour le marketing politique et territorial et la notoriété de la ville. L'adoption de la nouvelle signature est largement traitée et légitimée dans le BM. Les Gratte-Ciel s'imposent à la fois comme le symbole de l'unité du territoire et le lieu de l'exercice du pouvoir (hôtel de ville). Ainsi, raconter les Gratte-Ciel, c'est contribuer à une mémoire commune mais c'est aussi participer à la légitimité du pouvoir et d'une équipe municipale. La mémoire du centre-ville est fondamentalement une mémoire "par le haut ", institutionnelle, politique et idéologique. Elle ne se nourrit pas de la parole des habitants Sa valorisation est l'œuvre des acteurs institutionnels au pouvoir. Les interlocuteurs privilégiés pour l'évoquer jouissent d'une légitimité politique (parfois scientifique: historiens, sociologues...). Cette mémoire offre l'imaginaire politique et l'horizon utopique à partir desquels le lecteur est appelé à fonder sa citoyenneté.

\section{L'investissement mémoriel des quartiers}

19 En monopolisant une large part de l'investissement mémoriel, le centre-ville se donne comme le fondement identitaire du territoire villeurbannais. Mais s'il en fonde l'unité, il rompt, dans le même temps, la continuité urbaine en laissant apparaître une distinction centre/périphérie. Une mémoire politique à même de fonder la communauté est investie dans le centre-ville; des mémoires plurielles, elles, sont investies dans les quartiers périphériques. La question de la mémoire des populations urbaines « recèle des enjeux forts de visibilité, de reconnaissance, de légitimité pour les groupes concernés » (Chavanon, 2007, 63); elle s'inscrit, dans les BM, dans une problématique de la reconnaissance.

20 Villeurbanne est façonnée par l'histoire des migrations; et l'accueil offert aux migrants au long du $\mathrm{XX}^{\mathrm{e}}$ siècle constitue dans la communication municipale, et notamment dans les éditoriaux des maires villeurbannais, une véritable ressource identitaire. Un éditorial emblématique de Gilbert Chabroux, intitulé "Villeurbanne solidaire», illustre le mot d'ordre qui fonderait «l'esprit villeurbannais »: la solidarité. L'éditorial évoqué est diffusé au moment où la ville propose d'accueillir de jeunes Kosovars qui fuient une Yougoslavie déchirée : "S'il est quelques mots qui caractérisent notre ville avec ceux de l'audace, du courage, c'est bien le mot de solidarité [...]. Villeurbanne solidaire, c'est Villeurbanne qui accueille les réfugiés d'Arménie fuyant le génocide et l'extermination. C'est le Villeurbanne qui, 
entre deux guerres, donnait le nom Des Bienvenus à une de ses rues, pour accueillir les immigrés italiens et espagnols qui fuyaient les régimes nazis ou fascistes à l'œuvre dans leurs pays. Villeurbanne solidaire, c'est le Villeurbanne de toutes les générations, le Villeurbanne qui accepte ceux venus d'ailleurs pour qu'un plus soit apporté à notre cité» [éditorial, mai 1999]. La référence à la rue des Bienvenus est un cliché de la communication municipale. La rue inscrit dans les formes de l'espace les discours de la solidarité que valorisent les maires depuis 1990.

Dans les BM, le quartier est privilégié pour délimiter l'espace urbain. Il est à la fois une réalité fonctionnelle, un univers social, une pure représentation, un espace vécu (Tétu, 1995, 49). C'est une image, plus qu'une réalité, un espace déjà représenté et dans lequel s'inscrivent les pratiques et les mémoires. Il est en tout cas l'échelle privilégiée pour évoquer les mémoires plurielles et l'histoire des populations accueillies. Le quartier intervient dans les processus mémoriels dans un double sens : il est le repère stable qui permet au groupe de se souvenir et il porte les traces de son histoire.

Le registre mémoriel de «l'histoire vécue »- absent de l'évocation des Gratte-Ciel alimente la mémoire des quartiers. Cette dernière s'incarne dans la parole singulière et le témoignage. Pour autant, le parcours individuel se présente comme l'illustration d'une histoire collective : «La forme sémiotique du portrait modélise [...] les représentations associées à chaque communauté » en articulant "vies minuscules » qui " ne signifient rien d'autre qu'elles-mêmes " à "des vies exemplaires, conçues comme des modèles édifiés en fonction d'un système de valeur philosophique et moral» (Wrona, 2005, 95-108).

Les discours de la mémoire qui portent sur les quartiers s'inscrivent dans une problématique de la reconnaissance des diverses communautés qui composent aujourd'hui Villeurbanne. Ainsi, le quartier de la Poulette (originellement peuplé par les migrants italiens et espagnols) constitue le cadre territorial du souvenir des migrations italiennes. Ce quartier offre une consistance et donne à voir l'histoire d'une communauté. Les petites maisons, les jardins ouvriers... autant d'éléments d'urbanisme censés raconter la mémoire d'une communauté dont l'intégration est aujourd'hui perçue comme un modèle : «L'urbanisme montre aujourd'hui la façon dont ces ouvriers construisirent petit à petit leur maison, ajoutant des pièces au fil du temps... Chaque résident a pu concevoir son habitation selon ses goûts et ses moyens, si bien qu'on y trouve autant de modestes bicoques que de belles villas à étages » [Du côté de la Poulette, avril 1998].

Peu présents dans la mise en image du centre-ville (si ce n'est sous la forme d'une foule indistincte), les habitants intègrent largement la composition des photographies des quartiers. L'image relaie ainsi l'importance accordée à l'humain. Les témoignages et les récits de vie privilégient largement le registre affectif et évoquent une convivialité regrettée. Le rapport affectif à l'espace participe à la configuration du quartier comme territoire, support du souvenir et valeur refuge. Le récit des processions religieuses de Saint-Roch autour de l'église de la Sainte-Famille, rend compte du registre de discours privilégié : "Gisèle Matta, née Adamo, patronne de bar-fille d'Italiens : la foi et les pizzas : ‘[...] Petite fille, j'ai beaucoup fréquenté l'église de la Sainte-Famille, où j'ai fait ma communion. Je me souviens des fêtes de la Saint-Roch, début septembre. Le curé sortait la statue de Saint-Roch, il y avait une grande procession. On vendait des lupins et les pizzas que faisait ma mère sur le parvis. Toutes les familles italiennes du quartier se retrouvaient, c'était très gai, c'était vraiment la fête' » [L'église de la Sainte-Famille : le cœur des Italiens, mars 2003]. La dimension mémorielle 
du quartier rend compte, au risque parfois de les folkloriser, des cultures, des parcours, des traces de l'autre.

Reste que le registre de la nostalgie et l'attention portée aux parcours individuels et exemplaires participent aussi à voiler une réalité de l'immigration plus complexe et certainement plus conflictuelle. A ce titre, au contraire de la communauté italienne, la mémoire de la communauté algérienne semble souffrir de l'absence de lieux où s'incarner. Le lieu le plus emblématique de son histoire reste aujourd'hui, malgré sa disparition, le quartier Olivier de Serres. Né dans les années 1960, il abrite une population issue de l'immigration maghrébine (principalement algérienne). Les conditions de vie se dégradent très rapidement et, moins de vingt ans après, les premières barres sont détruites. Les dernières tombent en 1984. Le changement de nom illustre alors la volonté de tourner une page noire de la ville : Olivier de Serres devient Jacques Monod. Au large traitement médiatique accordé par le BM (et la presse régionale) aux différentes phases de la démolition, succède une longue période d'oubli qui illustre la difficulté de la ville à intégrer à son récit les épisodes les plus sombres de son histoire. La démolition constitue une violence symbolique, un déni de mémoire (Veschambre, 2008). En effet, les traces les plus sensibles d'une mémoire immigrée algérienne disparaissent avec le quartier et, avec elles, le souvenir des mauvaises conditions d'accueil alors offertes.

C'est à partir des années 1990 qu'une série d'initiatives (travaux de recherches, ouvrages de témoignages, expositions...) participe à l'émergence et à la valorisation d'une mémoire du quartier. La mémoire qui émerge s'inscrit dans une volonté de reconnaissance de la communauté maghrébine, de son histoire, de ses difficultés. Ces initiatives sont encore traitées a minima par le BM. Néanmoins, la trajectoire du souvenir olivier de Serres montre comment, à plus ou moins long terme, les discours peuvent venir compenser l'absence de traces et participer à une mémoire des lieux disparus. Elle illustre aussi la façon dont la mémoire se construit dans l'interaction entre acteurs sociaux : médiateur privilégié de l'identité villeurbannaise promue par l'équipe municipale, le BM ne jouit pas du monopole de la mémoire.

\section{Conclusion}

Les BM associent donc deux modes d'appréhension de l'espace urbain : une territorialité de nature politique et idéologique associée aux Gratte-Ciel ; et une territorialité vécue et affective - alimentée notamment par le récit de vie et le témoignage - qui est, elle, associée aux quartiers. Ces deux modalités d'appréhension de l'espace recouvrent une distinction centre/périphérie: un centre, lieu du politique où le pouvoir local et l'autonomie de la ville s'offrent au regard par la figure imposante des Gratte-Ciel et une périphérie traitée moins politiquement que culturellement. Le centre est le lieu de l'unité de la communauté et stimule le sentiment d'appartenance à la commune ; la périphérie, c'est-à-dire les quartiers, est le lieu de la reconnaissance des particularismes, qu'ils soient sociaux, culturels ou religieux. Dans l'un comme dans l'autre, la mémoire est mobilisée par les BM comme une ressource pour tisser du lien entre le passé et le présent, et instaurer un continuum entre mémoire(s) et territoire(s). La mémoire, dans les BM, maintient donc des liens symboliques propres à fonder, à la fois, l'identification à un destin collectif et la reconnaissance des parcours des différents groupes qui composent la communauté. 


\section{BIBLIOGRAPHIE}

Bonneville M., (1978), Villeurbanne : naissance et métamorphose d'une banlieue ouvrière. Processus et formes d'urbanisation, Lyon, PUL, $287 \mathrm{p}$.

Boullier B., (2010), La ville événement. Foules et publics urbains, Paris, PUF, 147 p.

Chavanon O., (2007), La folklorisation du souvenir des immigrés, in Gras P. et al., Ville et mémoire du voyage, pp. 63-73, Paris, L'Harmattan, 132 p.

Clemençon A.-S. (dir.), (2004), Les Gratte-Ciel de Villeurbanne, Lyon, Les Editions de l'imprimeur, $235 \mathrm{p}$.

Di Meo G., (1998), Géographie sociale et territoires, Paris, Nathan Université, 320 p.

Faure A., (2010), Banlieue, in Topalov C. et al., L'aventure des mots de la ville. A travers le temps, les langues, les sociétés, pp. 72-77, Paris, Robert Laffont, 1490 p.

Haas V. et Jodelet D., (2000), La mémoire, ses aspects sociaux et collectifs, in Roussiau N. (dir.), Psychologie sociale, pp. 121-134, Paris, Press Edition, 383 p.

Halbwachs M., (1950/1997), La mémoire collective, Paris, Albin Michel, 295 p.

Lamizet B. et Sanson P. (dir.), (1997), Les langages de la ville, Marseille, Ed. Parenthèses, 187 p.

Lamizet B., (2011), Le langage politique. Discours, images, pratiques, Paris, Editions Ellipses, 255 p.

Lamizet B., (2002), Le sens de la ville, Paris, L'Harmattan, 242 p.

Le Bart C., (2000), Les bulletins municipaux : une contribution ambiguë à la démocratie local, in Hermès, $n^{\circ}$ 26-27, pp. 175-184.

Lussagnet N., (1995), Photographies et temporalité dans la presse des collectivités locales, in Tétu J.F. (dir.), Ville et information, pp. 81-89, Lyon, Recherches en sciences humaines, 129 p.

Pailliart I., (2006), Territoires, identités et communication, in Olivesi S. (dir.), Sciences de l'information et de la communication. Objets, savoirs, disciplines, pp. 113-128, Grenoble, PUG, $286 \mathrm{p}$.

Raffestin C., (1980), Pour une géographie du pouvoir, Paris, Librairie Technique, 250 p.

Rhein C., (2010), « Ghetto », in Topalov C. et al., L'aventure des mots de la ville. A travers le temps, les langues, les sociétés, pp. 534-539, Paris, Robert Laffont, 1490 p.

Ricœur P., (2000), La mémoire, l'histoire, l'oubli, Paris, Le Seuil, 2000, 675 p.

Scherrer F., (1995), Genèse et métamorphose d'un territoire d'agglomération : de Lyon au Grand Lyon, in Revue de géographie de Lyon, vol. 70, n² 2, pp. 105-114.

Tétu J.-F. (dir.), (1995), Ville et information, Lyon, Recherches en sciences humaines, 129 p.

Vadelorge L., (2003), Des villes pour mémoire, in Ethnologie française, vol. 33, pp. 5-12.

Veschambre V., (2008), Traces et mémoires urbaines. Enjeux sociaux de la patrimonialisation et de la démolition, Rennes, PUR, $315 \mathrm{p}$.

Wrona A., (2005), Vies minuscules, vies exemplaires : récit d'individu et actualité, in Réseaux, $\mathrm{n}^{\circ} 132$, pp. 93-110. 


\section{NOTES}

1. Intitulé Bulletin municipal officiel de Villeurbanne et sur-titré «République Française. Liberté. Egalité. Fraternité » depuis 1960, le support se limite longtemps à quatre rubriques : "Conseil municipal ", «Lois, arrêtés et actes administratifs", " Avis, communications et informations diverses» et «Etat civil». En 1974, le BM initie sa mue avec un changement de format et un nouveau nom : Villeurbanne, notre ville. L'information se diversifie, les rubriques se multiplient: «Editorial», «Actualités villeurbannaises », «Reportages», «Pour mieux connaître Villeurbanne », etc. Le compte-rendu du conseil municipal est réduit à un tiers de page. En 1977, le BM devient Vivre à Villeurbanne; puis, en 1983, Aujourd'hui Villeurbanne. En 1985, le BM change de statut et la gestion revient à l'Office Villeurbannais d'Information et d'Expression (OVIDE). Depuis 1989, le BM est titré Viva.

2. 1944-1947 : Georges Lévy, PCF/1947-1954 : Lazare Goujon, SFIO/1954-1977 : Étienne Gagnaire MDSF/1977-1990 : Charles Hernu, PS/1990-1997 : Gilbert Chabroux PS/1997-1998: Raymond Terracher, PS/1998-2001 : Gilbert Chabroux PS/2001- : Jean-Paul Bret, PS.

\section{RÉSUMÉS}

Analysant la collection des Bulletins municipaux de Villeurbanne depuis 1974, cet article montre comment la mémoire contribue à l'identité de la ville, à la différenciation et à l'autonomie du territoire urbain. L'article envisage ainsi la distinction centre/quartiers dans les modalités d'investissement mémoriel du territoire et montre comment cette distinction illustre la tension entre la volonté de faire territoire (mémoire singulière du territoire) et la volonté de reconnaître aux quartiers leurs spécificités en admettant, ce faisant, la fragmentation de l'espace urbain (mémoire multiple du territoire).

Analyzing the collection of municipal reports in Villeurbanne (France) since 1974, this paper shows how memory contributes to the identity of the city and to the differentiation and autonomy of the urban territory. The paper considers the distinction between center and districts in the modalities of investment in memory in the territory, and shows how this distinction illustrates the tension between the will to make the territory (or the singular memory of the territory) and the will to grant districts their specificities by admitting the fragmentation of the urban space (or the multiple memory of the territory).

\section{INDEX}

Keywords : media, territory, identity, memory, municipal communication

Mots-clés : médias, territoire, identité, mémoire, communication municipale 


\section{AUTEURS}

\section{JULIEN AUBOUSSIER}

ELICO - Université de Lyon

Julien Auboussier, ELICO (Equipe lyonnaise en information et communication) EA 4147, Université de Lyon. Thèmes de recherche : médias, discours, espace public, territoires. Articles récents : « La dilution du discours antimondialisation dans les discours de presse », Mots. Les langages du politique, à paraître ; « Identités de club, territoires et imaginaires dans la PQR. Le derby Lyon-Saint Etienne », Les Cahiers du journalisme, 19, hiver 2009, pp. 130-142. Adresse électronique : auboussier_julien@hotmail.com.

\section{ISABELLE GARCIN-MARROU}

ELICO - Université de Lyon

Isabelle Garcin-Marrou, ELICO (Equipe lyonnaise en information et communication) EA 4147, Sciences Po Lyon, professeure en sciences de l'information et de la communication. Thèmes de recherche : médias, discours, violences, genre et territoires. Publications récentes : « Les émeutes de 2005 dans les discours de presse américains et français. Mémoires et diversité des regards médiatiques ", Thiéblemont S. et Koukoutsaki-Monnier A. (dirs.), Médias, dispositifs, médiations, PUN, 2010, pp. 121-140 ; " Ségolène Royal ou le difficile accès au panthéon politique », Mots. Les langages du politique, $\mathrm{n}^{\circ} 90$, juillet 2009, pp. 13-29. Adresse électronique : Isabelle.GarcinMarrou@sciencespo-lyon.fr. 